\title{
The extended counterpart of submm source Lockman $850.1^{\star}$
}

\author{
D. Lutz ${ }^{1}$, J. S. Dunlop 2 , O. Almaini ${ }^{2}$, P. Andreani ${ }^{6,1}$, A. Blain ${ }^{8}$, A. Efstathiou ${ }^{4}$, M. Fox $^{4}$, R. Genzel ${ }^{1}$, \\ G. Hasinger ${ }^{9,1}$, D. Hughes ${ }^{5}$, R. J. Ivison ${ }^{3}$, A. Lawrence ${ }^{2}$, R. G. Mann ${ }^{2}$, S. Oliver ${ }^{7}$, J. A. Peacock ${ }^{2}$,
} D. Rigopoulou ${ }^{1}$, M. Rowan-Robinson ${ }^{4}$, S. Scott ${ }^{2}$, S. Serjeant ${ }^{10}$, and L. Tacconi ${ }^{1}$

1 Max-Planck-Institut für extraterrestrische Physik, Postfach 1312, 85741 Garching, Germany

2 Institute for Astronomy, Department of Physics \& Astronomy, University of Edinburgh, Blackford Hill, Edinburgh EH9 3HJ, UK

3 UK ATC, Royal Observatory, Blackford Hill, Edinburgh EH9 3HJ, UK

4 Astrophysics Group, Imperial College London, Blackett Laboratory, Prince Consort Road, London SW7 2BZ, UK

${ }^{5}$ Instituto Nacional de Astrofisica, Optica y Electronica (INAOE), Apartado Postal 51 y 216, 72000 Puebla, Pue., Mexico

6 Osservatorio Astronomico di Padova, vicolo dell'Osservatorio 5, 35122 Padova, Italy

7 Astronomy Centre, University of Sussex, Falmer, Brighton BN1 9QJ, UK

8 Institute of Astronomy, University of Cambridge, Madingley Road, Cambridge CB3 0HA, UK

9 AIP, An der Sternwarte 16, 14482 Potsdam, Germany

10 Unit for Space Sciences and Astrophysics, University of Kent, Canterbury, Kent CT2 7NR, UK

Received 11 June 2001 / Accepted 6 August 2001

\begin{abstract}
The IRAM Plateau de Bure mm interferometer and deep $K$-band imaging have been used to identify the brightest submm source detected in the Lockman field of the UK 8 mJy SCUBA survey. The near infrared counterpart is an extended $(20-30 \mathrm{kpc})$, clumpy, and extremely red object. The spectral energy distribution suggests it to be a dusty star forming object at a redshift of about $3(2-4)$. Its star formation rate and nearinfrared properties are consistent with Lockman 850.1 being a massive elliptical in formation.
\end{abstract}

Key words. galaxies: active - galaxies: individual: Lockman 850.1 - galaxies: starburst - infrared: galaxies

\section{Introduction}

Over the past years, detections of (sub)mm sources in deep surveys have opened a new window on the high redshift universe (e.g. Smail et al. 1997; Hughes et al. 1998; Barger et al. 1998; Eales et al. 1999; Bertoldi et al. 2000). The source population discovered by SCUBA contributes a large fraction of the cosmic submm background. A significant part of star formation at redshifts 2 to 4 may be located in such dusty and extremely luminous galaxies. With inferred star formation rates of $\approx 1000 M_{\odot} \mathrm{yr}^{-1}$, they may indicate the formation of present day massive spheroids. A direct link between star formation/spheroid growth on one side and AGN fuelling/black hole growth on the other is not only plausible through gas fuelling

Send offprint requests to: D. Lutz, e-mail: lutz@mpe.mpg.de

* Based on Observations carried out with the IRAM Plateau de Bure Interferometer. IRAM is supported by INSU/CNRS (France), MPG (Germany) and ING (Spain). triggered by interactions or merging, but also suggested by the correlation between black hole and spheroid mass (Magorrian et al. 1998) and by global similarities in the cosmic evolution of star formation and quasar activity. In turn, this relates to the question of the starburst and AGN contribution to the power output of SCUBA sources, and their role in the origin of the cosmic X-ray and infrared backgrounds (e.g. Fabian et al. 2000; Hornschemeier et al. 2000)

Progress in all these issues relies on accurate identifications and redshift determinations, which are extremely difficult because of the large $\left(15^{\prime \prime}\right)$ SCUBA beam and the high density of very faint possible optical counterparts. A key step in this identification process is accurate astrometry. This can be obtained through mm interferometry, which directly locates the thermal dust emission (Downes et al. 1999; Bertoldi et al. 2000; Gear et al. 2000; Frayer et al. 2000). Such astrometry is demanded by the variance in optical/near-infrared properties of proposed 


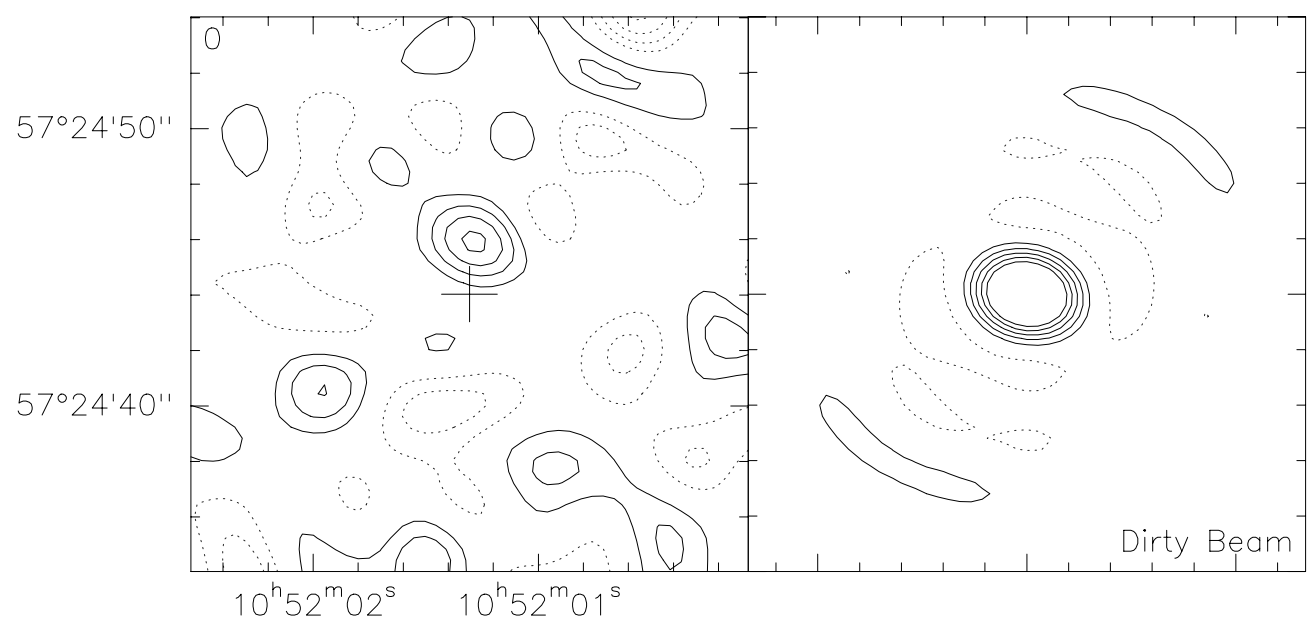

Fig. 1. IRAM Plateau de Bure 1.26 mm interferometric map of Lockman 850.1 (left, not CLEANed) along with the corresponding dirty beam including the sidelobes (right). Linear contours are spaced by $0.7 \mathrm{mJy}$. The cross denotes the phase center of the observation.

counterparts, which makes color-based identification criteria indecisive. Colors range from fairly blue (e.g. SMM02399, Ivison et al. 1998) to fainter very red objects (e.g. Smail et al. 1999; Gear et al. 2000; Bertoldi et al. 2000) and may include objects that are optically blank to the limit of the deepest HST images (Smail et al. 2000a; Muxlow et al. 2001). Accurate source positions and identifications also test hypotheses suggesting a local nature of part of the submm sources (Lawrence 2001).

The UK submm survey consortium is undertaking a wide field survey of $850 \mu \mathrm{m}$ sources with 3-sigma flux densities $>8 \mathrm{mJy}$. This survey is centred on the Lockman Hole (East), and the ELAIS N2 field, both previously surveyed with ISOPHOT at $175 \mu \mathrm{m}$ (Kawara et al. 2000; Lagache et al. 2001) and with the VLA (de Ruiter et al. 1997; Ciliegi et al. 1999). Spectral energy distribution (SED) arguments suggest that SCUBA sources detected neither by ISOPHOT nor in the moderately deep VLA data must lie at $z \gtrsim 1.5$. Lockman 850.1 is the brightest SCUBA source detected in the Lockman Hole part of this survey (Scott et al. 2001), a region extensively observed in X-rays (Hasinger et al. 1998, 2001).

\section{Millimeter interferometry}

In order to accurately locate the (sub)mm emission of Lockman 850.1, the source was observed with the IRAM Plateau de Bure (PdB) interferometer (Guilloteau et al. 1992) for a total of 25.9 hours on 1999 June 22, August 30, October 31, and November 5. Observations were done partly in the $5 \mathrm{D}$ and partly in the $4 \mathrm{D}$ configurations, with phase center at the nominal position from the SCUBA map (Table 1). Receivers were tuned to $238.46 \mathrm{GHz}(1.26 \mathrm{~mm})$ and $90.0 \mathrm{GHz}(3.33 \mathrm{~mm})$. The flux scale was calibrated using observations of $3 \mathrm{C} 273$ and $0923+392$. Temporal variations of amplitude and phase were calibrated by frequent observations of $0917+624$ and
1044+719. Data were reduced at IRAM Grenoble using GAG software. A positive signal is seen in all individual tracks. The final map shown in Fig. 1 was produced with natural weighting and has not been CLEANed.

We derived the total flux at $238 \mathrm{GHz}$ of $3.03 \pm$ $0.56 \mathrm{mJy}$, and the position listed in Table 1 from a point source fit to the visibilities. The positional uncertainty is obtained from adding in quadrature the statistical error of the fit and an astrometric uncertainty of $0.2^{\prime \prime}$ (see Downes et al. 1999). At the signal-to-noise of our data, no strong constraint can be put on the size of the $\mathrm{mm}$ source - the $1.26 \mathrm{~mm}$ map is consistent with the $2.8^{\prime \prime} \times 2.0^{\prime \prime}$ FHWM beam of our observations as well as with a centrally peaked structure extended on a few arcsec scale. No source was detected in the $3 \mathrm{~mm}$ band ( $3 \sigma$ upper limit $0.6 \mathrm{mJy}$ at $90 \mathrm{GHz}$ ), in agreement with the emission being due to dust.

We have obtained additional photometry of Lockman 850.1 on 2000 March 2 and March 5 using the MAMBO bolometer array at the IRAM $30 \mathrm{~m}$ telescope in on-off mode. The $11^{\prime \prime}$ beam was centered on the $\mathrm{PdB}$ interferometric position (Table 1). Averaging all 45 minutes of data we obtain a $1.2 \mathrm{~mm}$ flux of $3.8 \pm 0.5 \mathrm{mJy}$. In addition to the detection map, $850 \mu \mathrm{m}$ photometry was obtained with SCUBA in January 2000 confirming the detection with a flux of $10.3 \pm 2.1 \mathrm{mJy}$. A marginal detection is obtained from the SCUBA map at $450 \mu \mathrm{m}$. We adopt the mean of two independent reductions (Fox et al. 2001; Scott et al. 2001). Photometry of Lockman 850.1 is completed by a detection at $1.4 \mathrm{GHz}$ (Ivison et al. 2001a). The position of the radio detection is in excellent agreement with the mm detection (Table 1). In the $5 \mathrm{GHz}$ data of Ciliegi et al. (2001) a $2.5 \sigma$ maximum is seen, consistent with extrapolation from the $1.4 \mathrm{GHz}$ point assuming nonthermal emission. We list a $5 \mathrm{GHz}$ upper limit in Table 2 . 

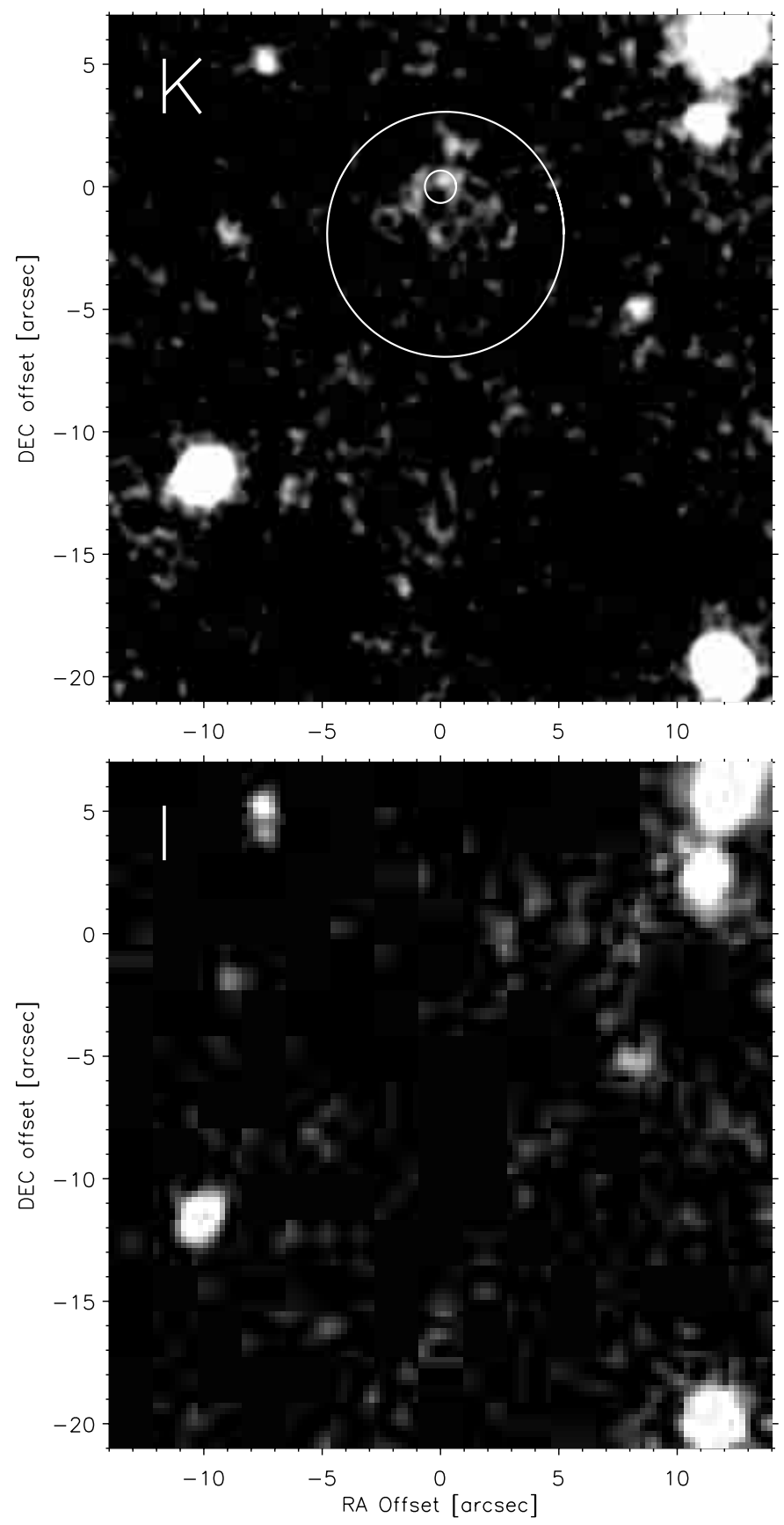

Fig. 2. Top: $K$ band image of the submm source Lockman 850.1. The big circle denotes a $5^{\prime \prime}$ uncertainty radius around the nominal SCUBA position, the small circle a $0.66^{\prime \prime}$ $(2 \sigma)$ uncertainty radius around the IRAM interferometric position. Offsets in arcsec are given with respect to the IRAM position. Bottom: $I$ band image of the same region, showing the nondetection of Lockman 850.1 at that wavelength.

\section{Optical/near-infrared imaging}

A deep $K$ band image of Lockman 850.1 was obtained in $246 \mathrm{~min}$ total integration time during the nights of May 17-21, 2000, using the UKIRT Fast Track Imager. The $K$-band seeing in the coadded data is $0.9^{\prime \prime}$. A knotty extended object is clearly detected at the position of the submm source (Fig. 2, Table 1), we list in Table 2
Table 1. Position of Lockman 850.1 (J2000). The positional errors do not include systematic offsets between the radio and optical (USNO A2) reference frame estimated at $\approx 0.3^{\prime \prime}$.

\begin{tabular}{lll}
\hline & RA $(\mathrm{J} 2000)$ & DEC $(\mathrm{J} 2000)$ \\
\hline SCUBA & $105201.31 \pm 2.5^{\prime \prime}$ & $+572444.0 \pm 2.5^{\prime \prime}$ \\
IRAM PdB & $105201.284 \pm 0.33^{\prime \prime}$ & $+572445.94 \pm 0.28^{\prime \prime}$ \\
$K$-band peak & $105201.26 \pm 0.3^{\prime \prime}$ & $+572446.2 \pm 0.3^{\prime \prime}$ \\
VLA 1.4 GHz & $105201.252 \pm 0.05^{\prime \prime}$ & $+572445.86 \pm 0.05^{\prime \prime}$ \\
\hline
\end{tabular}

Table 2. Photometry of Lockman 850.1.

\begin{tabular}{lrr}
\hline Band & Flux/Magnitude & Note \\
\hline $0.5-2 \mathrm{keV}$ & $<3 \times 10^{-16} \mathrm{erg} \mathrm{s}^{-1} \mathrm{~cm}^{-2}$ & Hasinger et al. (2001) \\
$m_{I}$ & $>26.0 \mathrm{mag}$ & in $4^{\prime \prime}$ diameter \\
$m_{I}$ & $>27.5 \mathrm{mag}$ & in $1^{\prime \prime}$ diameter \\
$m_{K}$ & $19.8 \pm 0.2 \mathrm{mag}$ & in $4^{\prime \prime}$ diameter \\
$m_{K}$ & $21.4 \pm 0.05 \mathrm{mag}$ & in $1^{\prime \prime}$ diameter \\
$S_{7 \mu \mathrm{m}}$ & $<0.2 \mathrm{mJy}$ & Fadda et al. (2001) \\
$S_{15 \mu \mathrm{m}}$ & $<0.2 \mathrm{mJy}$ & Fadda et al. (2001) \\
$S_{175 \mu \mathrm{m}}$ & $<200 \mathrm{mJy}$ & Estim. from Kawara 2000 \\
$S_{450 \mu \mathrm{m}}$ & $35.0 \pm 10 \mathrm{mJy}$ & SCUBA $8^{\prime \prime}$ beam \\
$S_{850 \mu \mathrm{m}}$ & $10.5 \pm 1.6 \mathrm{mJy}$ & SCUBA $15^{\prime \prime}$ beam \\
$S_{1.2 \mathrm{~mm}}$ & $3.8 \pm 0.5 \mathrm{mJy}$ & MAMBO 11" beam \\
$S_{1.26 \mathrm{~mm}}$ & $3.03 \pm 0.56 \mathrm{mJy}$ & PdB interferometer \\
$S_{3.33 \mathrm{~mm}}$ & $<0.6 \mathrm{mJy}$ & PdB interferometer \\
$S_{5 \mathrm{GHz}}$ & $<0.07 \mathrm{mJy}$ & Ciliegi et al. (2001) \\
$S_{1.4 \mathrm{GHz}}$ & $0.074 \pm 0.013 \mathrm{mJy}$ & Ivison et al. (2001a) \\
\hline
\end{tabular}

photometry for both the central knot and an aperture enclosing the extended $K$ band emission. No optical counterpart is detected in a deep $I$-band image taken at the WHT Prime Focus Imager (3500 s, 0.75" seeing, see Fig. 2) and in less deep $R$ and $V$ imagery. At $I-K>6.2$, Lockman 850.1 is a bona fide extremely red object (ERO), and a Class I SCUBA source in the terminology of Ivison et al. (2000b).

\section{Discussion}

Table 2 summarizes the available photometry of Lockman 850.1. The spectral energy distribution (SED) is shown in Fig. 3, along with comparison SEDs for which we have chosen the observed SED of Arp220, which has an extremely steep rest frame mid- to far-IR spectrum, and a schematic SED that is more typical for the properties of local ULIRGs. A first conclusion is that the SED of Lockman 850.1 is well described by an ultraluminous $\left(L_{\mathrm{IR}} \approx 8 \times 10^{12} L_{\odot}\right.$ for $\left.H_{0}=75 \mathrm{~km} \mathrm{~s}^{-1} \mathrm{Mpc}^{-1}, q_{0}=0.5\right)$ infrared galaxy at at redshift of around 3 .

The redshift of the source can be estimated on the basis of its mm-to-radio spectral index. Using the "mean" relation for the $350 \mathrm{GHz} / 1.4 \mathrm{GHz}$ spectral index derived by Carilli \& Yun (2000, see also http: //www.aoc.noao.edu/ ccarilli/alphaz.shtml), the index of 0.90 observed for Lockman 850.1 corresponds to a most probable $z=2.7$. Conservatively adopting their " $z_{+}$" relation and the $1 \sigma$ errors on the radio and 


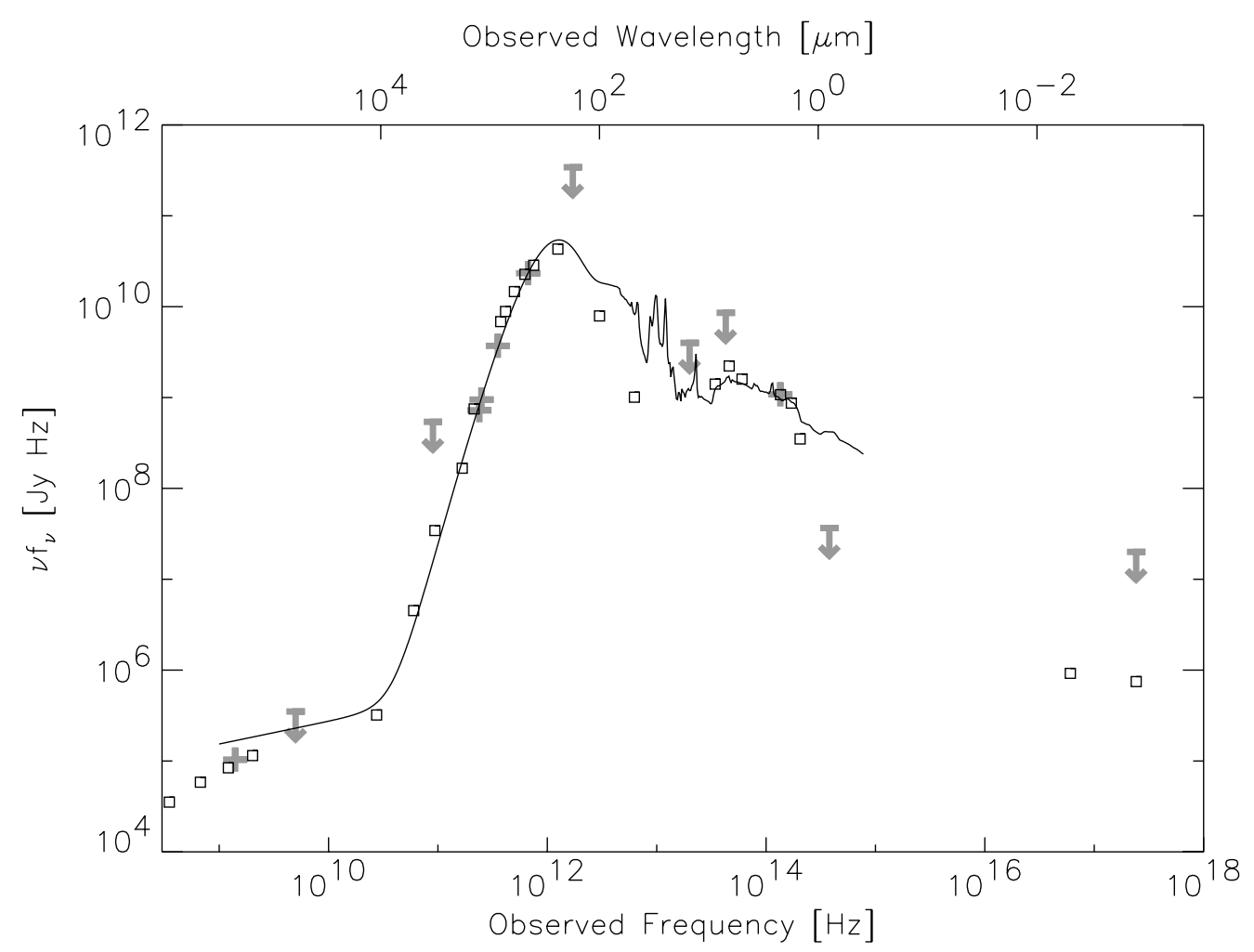

Fig. 3. Spectral energy distribution of Lockman 850.1 (thick grey crosses and limits). For comparison, we overplot the observed spectral energy distribution of Arp 220 scaled in flux and shifted to $z=3$ (small squares) and a schematic SED representing local ultraluminous galaxies, again shifted to $z=3$.
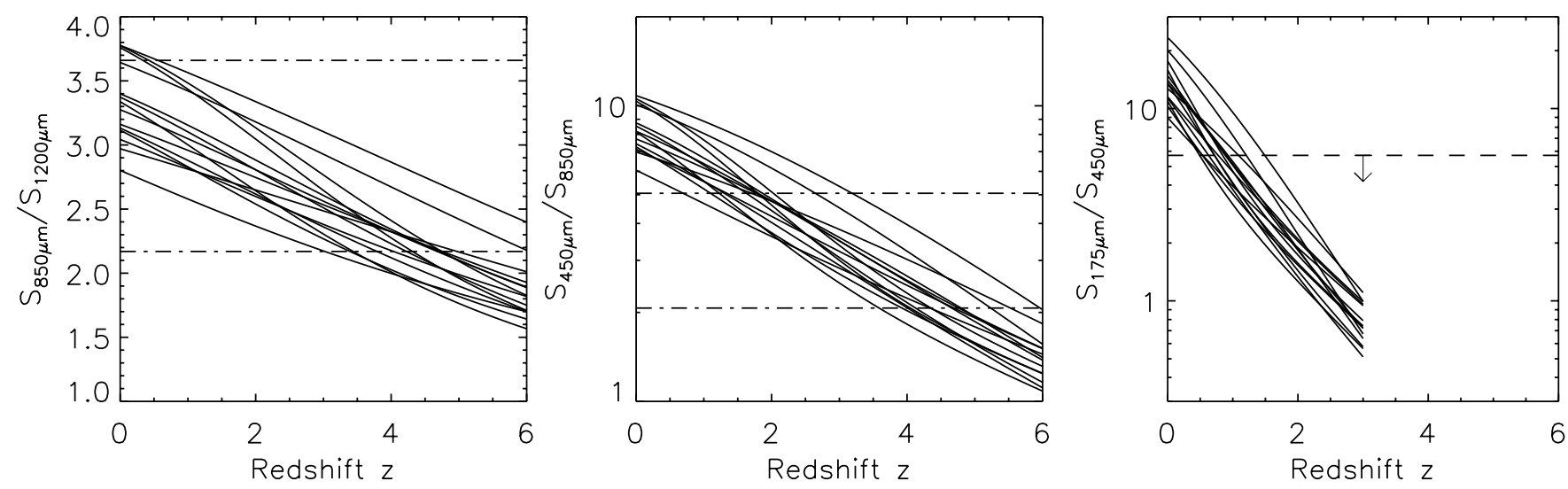

Fig. 4. Far-infrared photometric constraints on the redshift of Lockman 850.1. The continuous lines in each panel show the expected colors as a function of redshift predicted from the SEDs of 14 local ULIRGs from Klaas et al. (2001) that have detailed far-infrared ISO photometry as well as submm detections. Dashed lines indicate the allowed ranges or limits for Lockman 850.1.

submm flux the lower limit is $z=1.4$. Taking into account the flattening of the mm-to-radio spectral index curve for high redshifts and the variance of galaxy SEDs, the mm-to-radio index of Lockman 850.1 is consistent with all redshifts higher than these values but probably not above $\approx 6$ because of inverse Compton losses off the cosmic microwave background (Carilli \& Yun 1999). A very high redshift is also excluded by the submm SED. We have used far-infrared and submm photometry of local ULIRGs by Klaas et al. (2001) to assess constraints on the redshift of Lockman 850.1 and the uncertainty introduced by SED variance. Figure 4 shows the expected colors for those 14 objects in their sample which have both detailed far-infrared photometry out to $200 \mu \mathrm{m}$ and at least one submm detection. Their parametrization of the far-infrared/submm SEDs as single temperature modified blackbodies with finite optical depth is degenerate with alternative multiple temperature parametrizations. This does not affect Fig. 4, however, which just requires a good fit to the data. The predicted flux ratios shown in Fig. 4 change with redshift as rest wavelengths move from the Rayleigh-Jeans part of a modified blackbody 
curve through and beyond its peak. The large $850 \mu \mathrm{m}$ to $1.2 \mathrm{~mm}$ flux ratio is consistent with redshifts between 0 and $\approx 5$ taking into acount both the $1 \sigma$ flux errors and the $1 \sigma$ scatter in source redshifts for a given flux ratio. Recent observations of a fairly constant $450 \mu \mathrm{m}$ to $850 \mu \mathrm{m}$ flux ratio for local galaxies (Dunne \& Eales 2001) suggest that the parametrization chosen by Klaas et al. (2001) may overestimate the scatter in expected $850 \mu \mathrm{m}$ to $1.2 \mathrm{~mm}$ flux ratios for moderate redshift sources, but the flux errors and crosscalibration uncertainties remain a limitation for using this ratio. The $450 \mu \mathrm{m}$ to $850 \mu \mathrm{m}$ ratio for Lockman 850.1 suggests $z \approx 1.2 \ldots 5$, while the 175 to $450 \mu \mathrm{m}$ limit is not strongly constraining $(z>0.6)$. Further limits on the redshift are placed by the nondetection of Lockman 850.1 by ISOCAM at 7 and $15 \mu \mathrm{m}$. For an SED as shown in Fig. 3 the $15 \mu \mathrm{m}$ to $850 \mu \mathrm{m}$ index implies $z>2.1$. The SED of Arp 220 with its very low mid- to far-infrared flux ratio, however, would imply $z>1.5$. Overall, the variance of observed infrared/submm/radio SEDs of galaxies allows redshifts in the range 2 to 5 for Lockman 850.1. This is in good agreement with estimates we have obtained based on modelled SEDs (Efstathiou et al. 2000) that suggest $z=2 \ldots 4$ with a best guess of $z=3$.

The strong limit on the $I$ band flux, classifying Lockman 850.1 as an ERO, shows that the rest-frame optical/UV SED of Lockman 850.1 cannot be that of a "blue" starburst. The SED plotted in Fig. 3 assumes a ratio of infrared and blue luminosity representative for a ULIRG, but with a blue optical/UV continuum (scaling SED SB6 from Kinney et al. 1996). This is clearly inconsistent with the $I$ band limit. An intrinsically blue optical/UV SED would be possible for a very high redshift $I$ band dropout, but this is inconsistent with the submm SED. Trentham et al. (1999) have demonstrated that the slope of the optical/UV SEDs of local ULIRGs show large variations. A very steep, obscured SED like that of VII Zw 031 shifted to $z \sim 3$ matches the observations of Lockman 850.1.

Luminous infrared galaxies may be powered either by star formation or an AGN. The mid-infrared faintness of Lockman 850.1 is not suggestive of a strong AGN contribution. The XMM soft X-ray (rest frame hard X-ray) upper limit suggests $L_{2-10 \mathrm{kev}} / L_{\mathrm{bol}} \lesssim 10^{-3}$ and excludes an unobscured type $1 \mathrm{AGN}$. In the submillimetre to X-ray spectral index vs. redshift diagram of Fabian et al. (2000), the index limit of $>1.4$ places Lockman 850.1 even beyond Compton-thick obscured AGN with properties similar to NGC 6240, for all plausible redshifts. If Lockman 850.1 hosts an AGN, it must be weak and/or heavily obscured. The absence of an obvious central AGN in the $K$ image is consistent with these SED arguments.

The $K$ band morphology of Lockman 850.1 is clumpy and extended over about $4^{\prime \prime}$. It is clearly not that of a relaxed passive elliptical at intermediate redshift, as often found for (infrared-quiet) extremely red objects from near-IR surveys (Moriondo et al. 2000). On smaller scales, similar complexity has been observed in the $R$ band image of another SCUBA source, SMM 14011+0252
(Smail et al. 2000b, Ivison et al. 2001b). For a range of redshifts $(z=2 \ldots 5)$ and popular cosmologies $\left(H_{0}=\right.$ $65 \mathrm{kms}^{-1} \mathrm{Mpc}^{-1},\left(\Omega_{0}, \Omega_{\Lambda}\right)=(1,0)$ or $\left.(0.3,0.7)\right) 1^{\prime \prime} \approx$ $4 \ldots 8 \mathrm{kpc}$, i.e. Lockman 850.1 is a large system and even the individual clumps approach galactic scales. This morphology is more complex than the interacting galaxies associated with some other clearly identified (sub)mm sources (e.g. Smail et al. 1998; Ivison et al. 2000a; Bertoldi et al. 2000). Interacting non-merged systems are usually characterized by two nuclei and relatively faint tidal tails. More galaxies may be physically associated or projected, but the probability of obtaining a complex multi-peak morphology is small. The distinction between galaxy interactions and patchy star formation in a single large galaxy is a fuzzy one, of course, in the context of hierarchical buildup of large galaxies from smaller units. Lockman 850.1 does not resemble an interacting system in the specific meaning of an interaction of two well formed disk galaxies.

A non-negligible fraction of bright submm sources is expected to be lensed (Blain et al. 1999). There is no obvious candidate lens galaxy close to Lockman 850.1, however, and there are arguments against a model in which the brightest $K$ peak is the lens and the surrounding fainter clumps lensed images of the background submm source. The submm emission then would be associated with the fainter clumps around the lens only, and thus extended on a 4 arcsec scale without a central maximum. This is inconsistent with the compactness of the interferometric image, and the good agreement of interferometric and large beam (sub)mm fluxes. In addition, the nondetection in the $I$ band implies that the $I-K$ colors of all components must be extremely red, i.e. there is no evidence for a color difference between putative "lens" and "lensed object".

Most plausibly the clumps of Lockman 850.1 are part of a large and structured star forming galaxy, the clumps being individual very big star forming complexes and/or extinction minima in a patchy obscuration pattern seen against the rest frame optical/UV emission of the source. It has been argued on the basis of the huge inferred star formation rates $\left(\approx 1000 M_{\odot} \mathrm{yr}^{-1}\right.$ depending on assumptions about dust temperature and initial mass function) that submm galaxies highlight the formation of massive spheroids - these are exactly the star formation rates required to build a massive spheroid in a short time $(\sim 1 \mathrm{Gyr})$ if the formation is in one localised starburst. This scenario is strengthened by the consistency between the current mass in spheroids and the one inferred from IR/Submm counts (Tan et al. 1999). Lockman 850.1 may further support this by its morphology: the complex $K$ band morphology as well as the $K$ magnitude are reminiscent of $z>3$ radio galaxies (van Breugel et al. 1998; see also Pentericci et al. 2001), and radio galaxies in the local universe are known to reside in massive ellipticals. Strong dust emission observed in many high- $z$ radio galaxies (Archibald et al. 2001) is further evidence for similarities between submm sources and high- $z$ radio galaxies. Lockman 850.1 is also very luminous in the rest frame 
optical: adopting the estimated $z \approx 3$, it must be at least as bright as $M\left(B_{\text {rest }}\right) \approx-25$. Even taking into account evolutionary corrections, this suggests a very massive system. If the morphology of Lockman 850.1 reflects the buildup of an elliptical from $\approx 10 \mathrm{kpc}$ size clumps, observations of submm sources may constrain the cosmic time of these events in a hierarchical merger picture. Submm surveys may highlight the formation of some of today's massive ellipticals.

Acknowledgements. We are indebted to K. Meisenheimer for providing access to a CADIS $K$-band image of the Lockman hole region, and to U. Klaas and M. Haas for access to their ULIRG photometry. We thank D. Fadda and P. Ciliegi for information on unpublished mid-infrared and radio data. Special thanks go to the IRAM staff for excellent support during a difficult period.

\section{References}

Archibald, E. N., Dunlop, J. S., Hughes, D. H., et al. 2001, MNRAS, 323, 417

Barger, A. J., Cowie, L. L., Sanders, D. B., et al. 1998, Nature, 394, 248

Bertoldi, F., Carilli, C. L., Menten, K. M., et al. 2000, A\&A, 360, 92

Blain, A. W., Möller, O., \& Maller, A. H. 1998, MNRAS, 303, 423

Carilli, C. L., \& Yun, M. S. 1999, ApJ, 513, L13

Carilli, C. L., \& Yun, M. S. 2000, ApJ, 530, 618 and Erratum: 2000, ApJ, 539, 1024

Ciliegi, P., McMahon, R., Miley, G. K., et al. 1999, MNRAS, 302, 222

Ciliegi, P., et al. 2001, in preparation

de Ruiter, H. R., Zamorani, G., Parma, P., et al. 1997, A\&A, 319,7

Downes, D., et al. 1999, A\&A, 347, 809

Dunne, L., Eales, S. A. 2001, MNRAS, in press [astro-ph/0106362]

Eales, S. A., Lilly, S., Gear, W., et al. 1999, ApJ, 515, 518

Efstathiou, A., Rowan-Robinson, M., \& Siebenmorgen, R. 2000, MNRAS, 313, 734

Fabian, A. C., Smail, I., Iwasawa, K., et al. 2000, MNRAS, 315, L8

Fadda, D., et al. 2001, in preparation

Fox, M. J., et al. 2001, in preparation

Frayer, D. T., Smail, I., Ivison, R. J., \& Scoville, N. Z. 2000, AJ, 120, 1668
Gear, W. K., Lilly, S. J., Stevens, J. A., et al. 2000, MNRAS, 316, L51

Guilloteau, S., et al. 1992, A\&A, 262, 624

Hasinger, G., Burg, R., Giacconi, R., et al. 1998, A\&A, 329, 482

Hasinger, G., et al. 2001, A\&A, 365, L45

Hornschemeier, A. E., Brandt, W. N., Garmire, G. P., et al. 2000, ApJ, 541, 49

Hughes, D., Serjeant, S., Dunlop, J., et al. 1998, Nature, 394, 241

Ivison, R. J., Smail, I., Le Borgne, J.-F., et al. 1998, MNRAS, 298,583

Ivison, R. J., Smail, I., Barger, A. J., et al. 2000a, MNRAS, 315,209

Ivison, R. J., Dunlop, J. S., Smail, I., et al. 2000b, ApJ, 542, 27

Ivison, R. J., et al. 2001a, in preparation

Ivison, R. J., et al. 2001b, ApJ, submitted

Kawara, K., Sato, Y., Matsuhara, H., et al. 2000, in ISO Surveys of a Dusty Universe, ed. D. Lemke, M. Stickel, \& K. Wilke (Springer, Heidelberg), 49

Kinney, A., Calzetti, D., Bohlin, R. C., et al. 1996, ApJ, 467, 38

Klaas, U., Haas, M., Müller, S. A., et al. 2001, A\&A, submitted

Lagache, G., \& Dole, H. 2001, A\&A, 372, 702

Lawrence, A. 2001, MNRAS, 323, 147

Magorrian, J., Tremaine, S., Richstone, D., et al. 1998, AJ, 115,2285

Moriondo, G., Cimatti, A., \& Daddi, E. 2000, A\&A, 364, 26

Muxlow, T. W. B., Garrington, S. T., Richards, A. M. S., et al. 2001, in Proc. 5th EVN Symposium, in press

Pentericci, L., McCarthy, P. J., Röttgering, H. J. A., et al. 2001, ApJS, 135, 63

Scott, S., Fox, M., Dunlop, J. S., et al. 2001, MNRAS, submitted

Smail, I., Ivison, R. J., \& Blain, A. W. 1997, ApJ, 490, L5

Smail, I., Ivison, R. J., Blain, A. W., \& Kneib, J.-P. 1998, ApJ, 507, L21

Smail, I., Ivison, R. J., Kneib, J.-P., et al. 1999, MNRAS, 308, 1061

Smail, I., Ivison, R. J., Owen, F. N., et al. 2000a, ApJ, 528, 612

Smail, I., Ivison, R. J., Blain, A., \& Kneib, J.-P. 2000b, Proc. UMass/INAOE Conference "Deep Millimeter Surveys", in press [astro-ph/0008237]

Tan, J. C., Silk, J., \& Balland, C. 1999, ApJ, 522, 579

Trentham, N., Kormendy, J., \& Sanders, D. B. 1999, AJ, 117, 2152

Van Breugel, W. J. M., Stanford, S. A., Spinrad, H., Stern, D., \& Graham, J. 1998, ApJ, 502, 614 